\title{
Mudanças climáticas, mudanças produtivas e saúde: complexas interações na literatura nacional
}

\author{
Climate change, changes in productivity and health: \\ complex interactions in the national literature
}

Luciana Santos (https://orcid.org/0000-0002-0343-2592) ${ }^{1}$

Jessica Eloísa Oliveira (https://orcid.org/0000-0002-4549-6498) ${ }^{2}$

Alexandre Maduro-Abreu (https://orcid.org/0000-0001-8418-1803) ${ }^{3}$

Gabriela Litre (https://orcid.org/0000-0001-7789-0580) ${ }^{4}$

Guadalupe Souza Sátiro (https://orcid.org/0000-0001-5326-2707) ${ }^{5}$

Danny de Castro Soares (https://orcid.org/0000-0002-6665-9647) ${ }^{6}$

${ }^{1}$ Programa de PósGraduação em Bioética, Faculdade de Ciências da Saúde, Universidade de Brasília (UnB). Campus Universitário Darcy Ribeiro $\mathrm{s} / \mathrm{n}$, Asa Norte. 70910900 Brasília DF Brasil. lsantos213@hotmail.com ${ }^{2}$ Programa de Pós-

Graduação em

Administração,

Faculdade de Economia,

Administração e

Contabilidade, UnB. Brasília

DF Brasil.

${ }^{3}$ Centro de Pesquisa

em Gestão, Inovação

e Sustentabilidade,

Faculdade de Economia,

Administração e

Contabilidade, UnB. Brasília

DF Brasil.

${ }^{4}$ Centro de

Desenvolvimento

Sustentável, UnB. Brasília

DF Brasil.

${ }^{5}$ Programa de Pós-

Graduação em

Desenvolvimento

Sustentável, Centro

de Desenvolvimento

Sustentável, UnB. Brasília

DF Brasil.

${ }^{6}$ Programa de Pós-

Graduação em Economia,

Universidade Católica de

Brasília. Brasília DF Brasil.
Abstract This article reviews the scientific output published in national journals on the interactions that are established between the concepts of climate change, productive changes (including changes in land use) and human health in the last ten years (from 2008 to 2017), highlighting the theoretical-methodological trends of this output. To achieve this, a review of the literature was made from publications made available in the Scientific Electronic Library Online (SciELO) and the CAPES Journals Portal. The results obtained demonstrate the advances made in the last ten years by the interdisciplinary teams that dealt with the subject over the period analyzed. At the same time, this article identifies the research gaps that can be exploited in future research. In addition, a positive sophistication and refinement of analytical tools and instruments have been identified that are able to gather consistent information about a broad and complex reality characterized by the diversity of biomes, climatic scenarios and productive systems.

Key words Climate change, Changes in productivity, Human health, Review of the literature
Resumo Este artigo caracteriza a produção cientifica divulgada em periódicos nacionais sobre as interações que se estabelecem entre os conceitos de mudanças climáticas, mudanças produtivas (incluídas as mudanças no uso do solo) e saúde humana, nos últimos dez anos (de 2008 a 2017), com destaque para as tendências teórico-metodológicas dessa produção. Para tanto, realizou-se uma revisão da literatura a partir de publicações disponibilizadas nas bases Scientific Electronic Library Online (SciELO) e Portal de Periódicos CAPES. Os resultados obtidos demonstraram os avanços experimentados nos últimos dez anos pelas equipes interdisciplinares que se debruçaram sobre o tema ao longo do período analisado, bem como permitem identificar lacunas de pesquisa que podem ser exploradas em investigações futuras. Adicionalmente, foi identificada uma positiva sofisticação e refinamento das ferramentas e instrumentos analiticos que se mostram capazes de reunir informações consistentes sobre uma realidade ampla e complexa, caracterizada pela diversidade dos biomas, cenários climáticos e sistemas produtivos.

Palavras-chave Mudanças climáticas, Mudanças produtivas, Saúde humana, Revisão da literatura 


\section{Introdução}

O tema das mudanças climáticas e suas interações com as mudanças produtivas e a saúde humana tem de modo progressivo ganhado relevância nos debates, estudos e políticas públicas ${ }^{1-3}$. Na última década, entretanto, verifica-se no Brasil o aprofundamento da discussão sobre o tema, particularmente no meio acadêmico e no contexto das instituições públicas de pesquisa e ensino, onde o aperfeiçoamento de modelos de análise e diagnóstico, aliado ao desenvolvimento de sistemas de informação integrados, permitiram a espacialização temporal das vulnerabilidades que cada região do país apresenta. A este respeito, destacase a preocupação dos autores em compreender, desde uma perspectiva comparativa, as especificidades locais e os contrastes regionais existentes.

O presente artigo caracteriza a produção nacional sobre as interações que se estabelecem entre os conceitos de mudanças climáticas, mudanças produtivas (incluídas as mudanças no uso do solo) e saúde humana, no período de 2008 a 2017, em periódicos classificados entre A1 e B4 pela Coordenação de Aperfeiçoamento de Pessoal de Nível Superior (CAPES). A partir de filtros previamente escolhidos para a realização deste trabalho, foram selecionados 41 artigos cujas temáticas versavam sobre as interações entre mudanças climáticas, mudanças produtivas e saúde. Esses artigos científicos são oriundos de duas bases de dados, a Scientific Electronic Library Online (SciELO) e o Portal de Periódicos CAPES. No caso da plataforma SciELO, como será detalhado na metodologia, a primeira busca apresentou um total de 54 publicações. No Portal de Periódicos CAPES obtevese 314 artigos como resultado inicial, totalizando 368 estudos. A partir da leitura dos resumos e dos critérios de inclusão previamente definidos foram eliminados 298 artigos e, posteriormente, outras 29 publicações. Logo, a amostra deste estudo foi composta por 41 artigos tabulados e analisados por meio de estatística descritiva.

Desse conjunto, um primeiro ponto a ser observado se refere ao destaque dado pelos autores ao conceito de vulnerabilidade. Neste ponto, é possível aventar que, por seu caráter multifatorial e multifacetado, este conceito é apresentado nas análises a partir de diferentes graus de elaboração teórica, revelando-se um recurso precioso para composição do caleidoscópio de cenários e interações que se estabelecem entre mudanças climáticas, mudanças produtivas e saúde.

Ademais, há de se destacar a forma como os estudos na área focalizada tem incorporado, a partir de uma análise contextual, os avanços teóricos alcançados nos últimos anos por investigações produzidas sobre o conceito de vulnerabilidade, ao contemplarem em suas análises dimensões históricas, econômicas, sociais e institucionais em detrimento de aspectos exclusivamente biofísicos. De fato, essa tendência na literatura sobre o conceito de vulnerabilidade revela a emergência de novos enfoques para se tratar as suscetibilidades humanas diante dos desafios ambientais que se apresentam na atualidade ${ }^{4-6}$. Nesse contexto, em que se verifica a impossibilidade de estabelecer uma relação direta de causa e efeito entre mudanças climáticas, mudanças produtivas e condições de saúde, o cenário com o qual o pesquisador se depara se revela dinâmico, construído a partir de interações que só podem ser descritas por meio de uma teia de relações complexas e de origem multicausal.

Seguindo essa direção, a revisão dos artigos selecionados indica de que maneira uma abordagem multidimensional e interdisciplinar da vulnerabilidade se espelha na produção científica brasileira. Além de possibilitar a análise da abordagem teórico-metodológica a respeito de estratégias adaptativas que se revelam como forma de adequação dos grupos humanos aos novos cenários de mudanças climáticas e contextos produtivos no país.

De modo complementar, como se verá mais adiante, a análise da produção científica sobre os conceitos de mudanças climáticas, mudanças produtivas e saúde permite entrever a existência de um campo de estudos pautado pelo esforço crescente em definir novos modelos analíticos e instrumentos de pesquisa com potencial para descrever interações complexas, bem como prover cenários futuros. Frequentemente, o modelo analítico identificado na produção científica em questão foi além das reflexões teóricas sobre a vulnerabilidade e revelaram trabalhos de teor normativo, constituindo subsídios significativos para a formulação de políticas públicas e para a tomada de decisão em diferentes escalas, em especial no setor saúde.

A questão que orienta o presente trabalho remete às possibilidades e limites de uma abordagem conjunta de problemas relacionados às mudanças climáticas e produtivas e os efeitos sobre a saúde humana, particularmente, em relação aos aspectos teórico-metodológicos e instrumentos e modelos de análise utilizados pelos pesquisadores. Adicionalmente, trata-se de identificar lacunas de pesquisa como forma de prover subsídios para a formulação de estudos futuros. Para tanto, 
recorremos à revisão da literatura enquanto modelo de análise adequado à proposta e às questões que se apresentaram ao longo da construção deste artigo, considerando a relevância de estudos desse tipo para o dimensionamento da produção sobre um determinado tema ${ }^{7}$.

O presente trabalho está organizado em quatro partes, que incluem a Introdução, Procedimentos Metodológicos, Resultados e Considerações Finais.

\section{Procedimentos metodológicos}

Esta revisão de literatura caracteriza-se como descritiva, com abordagem mista (qualitativaquantitativa), na qual se realizou uma pesquisa documental em periódicos nacionais por meio das bases Scientific Electronic Library Online (SciELO) e Portal de Periódicos CAPES. O recorte foi longitudinal, ao considerar os artigos científicos publicados em periódicos nacionais no período de 2008 a 2017. As palavras-chave utilizadas para a pesquisa nas bases de dados foram mudanças climáticas, mudanças produtivas e saúde que, por sua vez, foram combinadas com operadores booleanos. A Figura 1 sintetiza as etapas seguidas para atingir o objetivo do artigo.
A primeira etapa do trabalho teve início em maio de 2018, quando se realizou a pesquisa nas bases de dados com todas as palavras-chave separadas pelo operador booleano and. Assim, a primeira busca foi feita da seguinte forma: "mudanças climáticas" AND "mudanças produtivas" AND saúde. Como essa busca inicial não apresentou nenhum resultado nas bases de dados, optou-se por trocar o operador booleano entre mudanças climáticas e mudanças produtivas para or, ficando a busca da seguinte maneira: ("mudanças climáticas" OR "mudanças produtivas") AND saúde. Na base SciELO foi possível realizar a pesquisa utilizando-se as três palavraschave em uma única busca, obtendo-se como resultado 54 publicações. A estrutura do Portal Periódicos CAPES, por outro lado, só permite incluir até duas palavras-chave por vez, o que fez com que a pesquisa fosse dividida em duas buscas distintas. Além do filtro de ano de publicação, no Portal Periódicos CAPES foi incluído o filtro "periódicos revisados por pares", o que levou a um resultado de 314 artigos. Ao final da primeira fase alcançou-se uma amostra inicial de 368 artigos nas duas bases de dados.

Os resumos dos 368 artigos anteriormente selecionados foram revisados na segunda etapa a partir de três critérios de inclusão: (1) possuir

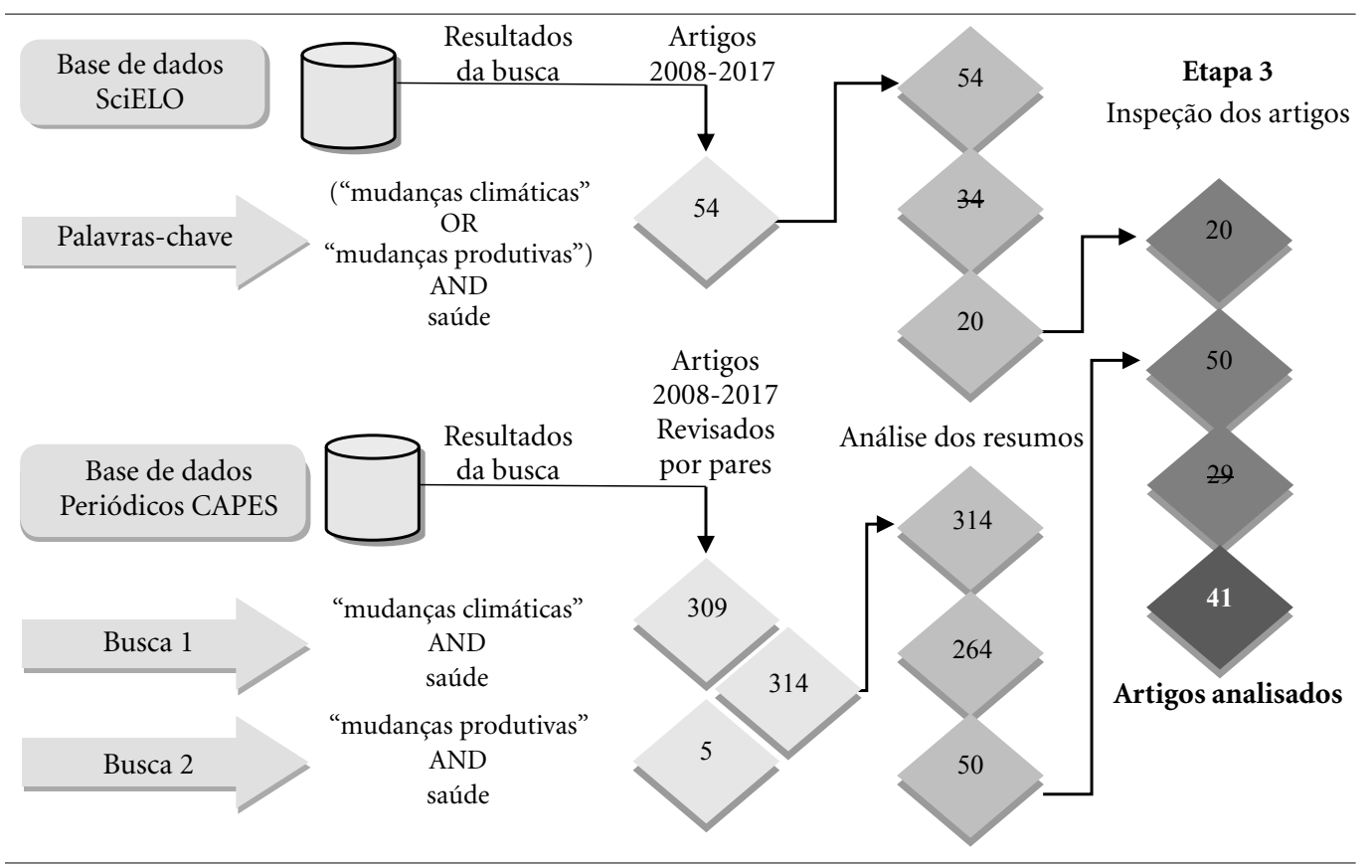

Figura 1. Protocolo da revisão de literatura. 
aderência ao tema do estudo; (2) ser publicado em um periódico nacional; e (3) ter como escopo o Brasil. Nesta etapa foram eliminados 298 artigos que não se enquadraram adequadamente aos critérios de inclusão. A maioria dos artigos eliminados, apesar de conter as palavras-chave pesquisadas (mudanças climáticas, mudanças produtivas e saúde), não integrava os temas de pesquisa ou apenas citavam as palavras-chave no decorrer da publicação. Outros artigos excluídos tratavam da integração dos temas de pesquisa em outros países ${ }^{8}$ ou foram publicados em periódicos internacionais ${ }^{9,10}$. Ao final da segunda etapa obteve-se um total de 70 artigos.

Na terceira fase realizou-se a análise dos artigos das duas bases de dados (Periódicos CAPES e SciELO) em conjunto. Assim, verificou-se que 12 artigos estavam incluídos de forma simultânea nas duas bases de dados. Logo, procedeu-se a eliminação das duplicidades. Na sequência, realizou-se a leitura das 58 produções restantes, ocasião em que se averiguou se os artigos atendiam a todos os critérios de inclusão. Nesta etapa, além dos 12 artigos em duplicidade, foram excluídos outros 17 de acordo com os critérios de inclusão citados anteriormente. Ao final, 41 artigos foram selecionados para análise.

Os artigos selecionados foram analisados e tabulados considerando-se três dimensões, a saber: (1) quadro geral das publicações; (2) perfil dos autores e aspectos metodológicos; e (3) finalidade temática da produção nacional. Em relação à primeira dimensão, foram extraídos dados gerais dos artigos, como título da revista, Qualis-CAPES e ano de publicação. A segunda dimensão buscou dados referentes aos autores (números de autores por artigo, área de formação, base, titulação máxima, filiação institucional e gênero) e aos aspectos metodológicos (abordagem de pesquisa, método, instrumento e técnicas analíticas). Nos casos em que os dados dos autores não estavam disponíveis nos artigos, foram consultados os currículos Lattes dos autores e/ou as instituições às quais eles estavam filiados de forma a complementar as informações. Finalmente, a terceira dimensão buscou identificar a finalidade temática dos artigos por meio da rede de palavras-chave, tomando-se os temas mais citados pelos autores e os subtemas a eles relacionados, considerando-se para este fim as palavras-chave, biomas estudados, principais indicadores de cada tema (saúde, mudanças climáticas e produtivas), vulnerabilidades e doenças mais citadas na produção nacional sobre o assunto estudado. A análise dos dados tabulados foi realizada por meio de estatística descritiva.

\section{Resultados}

Como mencionado, este estudo focalizou três dimensões de análise da literatura: (1) quadro geral das publicações; (2) perfil dos autores e aspectos metodológicos; e (3) finalidade temática da produção nacional. A seguir, apresentar-se-á os resultados da análise sobre os 41 artigos selecionados.

\section{Quadro geral}

Como se pode observar na Tabela 1, o trabalho de caracterização das fontes de publicação revela que a maioria dos artigos elegidos nessa investigação foi divulgada na Revista Ciência \& Saúde Coletiva (C\&SC) (43,90\%), periódico criado em 1996 e editado pela Associação Brasileira de Saúde Coletiva (Abrasco), com Qualis/ Capes A1 na área de Ensino. Com relação ao fator de impacto (FI), este periódico alcançou o índice de 0,780 , segundo a classificação do Journal Citation Reports (JCR), da Web of Science, além de ocupar o $1^{\circ}$ lugar no ranking do Google Acadêmico (Google Scholar em inglês), quando considerado todos os periódicos científicos brasileiros de qualquer área. Notadamente, os dados obtidos nesta pesquisa demonstram a importância desta revista para o intercâmbio e difusão de informações sobre o tema aqui focalizado, mostrando-se como a escolha preferencial dos autores para a divulgação dos seus trabalhos.

De forma menos expressiva, aparecem outros periódicos entre os quais é possível relacionar a Revista de Saúde Pública (RSP), editada pela Universidade de São Paulo e com Qualis/Capes A1, com 7,32\% da produção sobre o tema; e as revistas Saúde e Sociedade (SAUSOC), da Faculdade de Saúde Pública, Universidade de São Paulo e Associação Paulista de Saúde Pública, Qualis/ Capes A1; Revista Ra'e Ga: O Espaço Geográfico em Análise (RAEGA), do Departamento de Geografia e pelo Programa de Pós-Graduação em Geografia da Universidade Federal do Paraná, A2; e Revista Ambiente \& Água (AMBIAGUA), editada pelo Instituto de Pesquisas Ambientais em Bacias Hidrográficas, A2; ambas abrigando $4,88 \%$ dos artigos aqui elegidos.

No que diz respeito à classificação Qualis do conjunto de revistas, a pesquisa revelou que $65,85 \%$ dos artigos foram publicados em periódicos A1, conforme os critérios definidos pela Coordenação de Aperfeiçoamento de Pessoal de Nível Superior (CAPES); 17,7\% A2; 14,63\% B1 e, por fim, 2,44\% B4. Esse resultado revela o 
Tabela 1. Número e percentual de artigos por revista.

\begin{tabular}{clcr}
\hline \multicolumn{1}{c}{ Título (sigla) } & $\begin{array}{c}\text { Artigos } \\
\text { publicados } \\
\text { sobre o tema }\end{array}$ & \% \\
\hline $1413-8123$ & Revista Ciência \& Saúde Coletiva (CSC) & 18 & 43,90 \\
$0034-8910$ & Revista de Saúde Pública (RSP) & 3 & 7,32 \\
$0104-1290$ & Saúde e Sociedade (SAUSOC) & 2 & 4,88 \\
$2177-2738$ & Revista Ra'e Ga: O Espaço Geográfico em Análise (RAEGA) & 2 & 4,88 \\
$1980-993 X$ & Revista Ambiente \& Água (AMBIAGUA) & 2 & 4,88 \\
$1414-753 X$ & Revista Ambiente \& Sociedade (ASOC) & 1 & 2,44 \\
$0103-4014$ & Estudos Avançados (EA) & 1 & 2,44 \\
$0378-1844$ & Revista Interciencia (INTERCIENCIA) & 1 & 2,44 \\
$0102-3098$ & Revista Brasileira de Estudos de População (RBEPOP) & 1 & 2,44 \\
$1676-0611$ & Biota Neotropica (BN) & 1 & 2,44 \\
$0037-8682$ & Revista da Sociedade Brasileira de Medicina Tropical (RSBMT) & 1 & 2,44 \\
$0103-1104$ & Saúde em Debate (SDEB) & 1 & 2,44 \\
$1807-1600$ & HOLOS & 1 & 2,44 \\
$1679-4974$ & Epidemiologia e Serviços de Saúde (RESS) & 1 & 2,44 \\
$0100-204 X$ & Pesquisa Agropecuária Brasileira (PAB) & 1 & 2,44 \\
$0103-7331$ & Physis: Revista de Saúde Coletiva (PHYSIS) & 1 & 2,44 \\
$1980-1726$ & Hygeia: Revista Brasileira de Geografia Médica e da Saúde (HYGEIA) & 1 & 2,44 \\
$0104-4230$ & Revista da Associação Médica Brasileira (RAMB) & 1 & 2,44 \\
$2358-3592$ & Revista Política e Planejamento Regional (RPPR) & 1 & 2,44 \\
Total & & 41 & 100,00 \\
\hline
\end{tabular}

Fonte: Elaborado pelos autores.

destaque alcançado pelo tema nas revistas mais bem qualificadas. É preciso indicar que, embora tenham sido considerados os índices JCR e SCIMAGO, os resultados obtidos demonstram que a aplicabilidade desses critérios à qualificação da produção analisada é inviabilizada pelo fato de que um número inexpressivo dos periódicos listados dispunha deste tipo de classificação.

Em relação à distribuição das publicações ao longo do período focalizado, observa-se uma oscilação na frequência da produção científica sobre o tema, ao contabilizar 3 publicações em 2008; 1 em 2009; 1 em 2010; 2 em 2011; 6 em 2012; 1 em 2013; 8 em 2014; 2 em 2015; 13 em 2016 e, finalmente, um total de 4 publicações em 2017.

\section{Perfil dos autores}

Ao considerar os aspectos demográficos e a qualificação dos autores (Tabela 2), os resultados obtidos revelam que a distribuição mantém certo equilíbrio entre arranjos com dois $(21,95 \%)$ e três autores (19,51\%), seguidos da composição com cinco autores $(17,07 \%)$. Entretanto, foi possível observar como investigações mais complexas, que exigiram o aporte de dados mais especializados para sua elaboração, chegaram a contar com até onze pesquisadores para sua realização, o que por sua vez, revela a crescente tendência (já estabelecida no nível internacional ${ }^{11}$ a enxergar temas complexos desde uma lente interdisciplinar e por meio de redes de pesquisa colaborativas.

A classificação por gênero revela um dado interessante, quando se considera os 181 autores responsáveis pelas publicações, ao não apontar diferenças expressivas entre o número de publicações produzidas por homens $(47,51 \%)$ e mulheres $(52,49 \%)$ o que, por sua vez, aponta para uma convergência com os dados gerais das pesquisas realizadas no país, como sugerem os dados obtidos pela Gender in the Global Research Landscape $e^{12}$, que indicam que entre 2011 e 2015, $49 \%$ dos trabalhos científicos publicados no Brasil foram elaborados por mulheres.

Com relação à qualificação dos autores, à época da publicação do artigo, os dados revelam que, em sua maioria, os pesquisadores possuem qualificação máxima, ou seja, o título de doutorado $(67,40 \%)$, seguidos daqueles com título de mestrado (26,52\%); dado que demonstra o preparo desses pesquisadores para dirigir estu- 
dos de fôlego (Tabela 2). Por fim, as informações referentes à caracterização dos autores por área de formação (graduação) indicam um alto grau de dispersão dos dados, mesmo que se possa verificar um pequeno destaque para os graduados nas áreas de Geografia (12,71\%), Ciências Biológicas $(12,15 \%)$ e Engenharias (10,50\%), sem que se possa identificar a predominância de uma área específica. Entretanto, essa configuração reforça o fator da interdisciplinaridade como elemento chave para descrever as equipes de trabalho formadas sobre o tema, como apontado anteriormente.

Ao considerar os dados referentes apenas ao primeiro autor dos artigos, nota-se que há algumas distinções em relação às informações do conjunto total de autores. Neste caso, o perfil dos autores indica a predominância do gênero masculino (56,10\%), com doutorado $(58,54 \%)$ e formação variada, com destaque para as áreas de Geografia (14,63\%), Ciências Biológicas $(12,20 \%)$, Engenharias $(9,76 \%)$ e Enfermagem $(9,76 \%)$.

Outro elemento que merece ser destacado diz respeito à filiação institucional dos autores. De modo particular, é possível entrever a existência de uma dinâmica de grupos de trabalho e inter- câmbio de informações e dados entre instituições de pesquisa e ensino e órgãos do Governo Federal e que se revela enquanto uma característica relevante das equipes de investigação. Os 181 autores que publicaram os artigos estão filiados a 46 instituições diferentes, sendo que 11 instituições representam $66,85 \%$ dos autores. As demais 35 instituições agregam $33,15 \%$ dos autores. Nesse sentido, merece ênfase o papel de instituições como a Fundação Oswaldo Cruz (Fiocruz), que é representada por 54 dos autores responsáveis pelas investigações consideradas; o Ministério da Saúde, com 15 autores filiados, a Universidade Federal de Minas Gerais com 9 e, finalmente, a Universidade de São Paulo com 8.

Nessa direção, mencionam-se os intercâmbios existentes entre as diferentes instituições relacionadas nesta pesquisa, particularmente, a partir da colaboração entre grupos de trabalho e a existência de projetos de pesquisa compartilhados, caso exemplar do Observatório Nacional do Clima e Saúde (Fiocruz) e do Projeto Vulnerabilidade à Mudança do Clima (concebido pela Fiocruz em parceria com o Ministério do Meio Ambiente), assim como os estudos conduzidos pelo CEDENPLAR (UFMG) em colaboração com a Fiocruz.

Tabela 2. Perfil dos autores.

\begin{tabular}{|c|c|c|c|c|c|}
\hline & No & $\%$ & & No & $\%$ \\
\hline Número de autores & & & Áreas de formação (graduação) & & \\
\hline Um autor & 3 & 7,32 & Geografia & 23 & 12,71 \\
\hline Dois autores & 9 & 21,95 & Ciências Biológicas & 22 & 12,15 \\
\hline Três autores & 8 & 19,51 & Engenharia & 19 & 10,5 \\
\hline Quatro autores & 4 & 9,76 & Economia & 15 & 8,29 \\
\hline Cinco autores & 7 & 17,07 & Enfermagem & 12 & 6,63 \\
\hline Seis autores ou mais & 10 & 24,40 & Fisioterapia & 10 & 5,52 \\
\hline Total & 41 & 100 & Medicina & 9 & 4,97 \\
\hline Gênero dos autores & & & História & 8 & 4,42 \\
\hline Masculino & 86 & 47,514 & Medicina Veterinária & 7 & 3,87 \\
\hline Feminino & 95 & 52,486 & Estatística & 6 & 3,31 \\
\hline Total & 181 & 100 & Matemática & 5 & 2,76 \\
\hline Titulação máxima & & & Física & 4 & 2,21 \\
\hline Doutorado & 122 & 67,4 & Meteorologia & 4 & 2,21 \\
\hline Mestrado & 48 & 26,52 & Nutrição & 4 & 2,21 \\
\hline Especialização & 2 & 1,1 & Administração & 3 & 1,66 \\
\hline Graduação & 7 & 3,87 & Tecnologia Ambiental & 3 & 1,66 \\
\hline Aluno de graduação & 1 & 0,55 & Outras áreas & 23 & 12,71 \\
\hline Não informado & 1 & 0,55 & Não informado & 4 & 2,21 \\
\hline Total & 181 & 100 & Total & 181 & 100 \\
\hline
\end{tabular}

Fonte: Elaborado pelos autores. 


\section{Aspectos metodológicos}

No que concerne aos aspectos metodológicos, os trabalhos produzidos na interface entre mudanças climáticas, mudanças produtivas e saúde tem como desafio lidar com contextos diversos em que as relações de causa e efeito não se expressam de forma clara, mas a partir de interações complexas que exigem a elaboração de desenhos de pesquisa capazes de descrever a complexidade dos cenários climáticos e contextos produtivos.

A tipificação metodológica das investigações mostra que, em sua maioria, os autores adotaram uma abordagem quantitativa para tratar o tema $(58,54 \%)$, enquanto que $29,27 \%$ partem de uma perspectiva qualitativa e, por fim, $12,20 \%$ utilizam métodos mistos de análise (Tabela 3). No que toca à origem dos dados, verifica-se que grande parte das informações provém da pesquisa documental (58,54\%); 9,76\% são obtidas a partir de estudos de caso, sendo este mesmo registro obtido para dados de origens múltiplas e, por último, $2,44 \%$ dos dados utilizados nas pesquisas são produzidos a partir do trabalho etnográfico.

Ainda sobre o aspecto metodológico, os recursos adotados pelos pesquisadores são as estatísticas descritivas e inferenciais que alcançaram a mesma importância no conjunto, $21,95 \%$ cada uma; destacando-se, ainda, a estatística combina$\mathrm{da}$, que representa $19,51 \%$ do total de artigos analisados. Como se pode notar, particularmente em relação aos métodos de pesquisa e instrumentos de análise, as evidências permitem afirmar que a produção focalizada neste artigo privilegia a descrição conjunta das dinâmicas climáticas e dados sobre as condições de saúde, sem que sejam realizadas grandes elaborações teóricas sobre o tema. De modo geral, o que se verifica é a centralidade dos conceitos de vulnerabilidade e risco, ainda que sem maiores problematizações teóricas.

No mais, a ênfase parece incidir no esforço de constituição de bancos de dados e informações integrados que se mostraram, por sua vez, como o elemento de distinção e refinamento das pesquisas produzidas na área. Não obstante sejam mencionadas dificuldades no processo de desenvolvimento de modelos analíticos e instrumentos de pesquisa mais adequados à realidade das mudanças climáticas, bem como a necessidade de ampliar a disponibilidade e integração dos bancos de dados e informações existentes ${ }^{13}$, alguns avanços nessa área podem ser identificados.

A este respeito, nota-se como a partir do estabelecimento do SUS, em 1988, o setor saúde concentrou esforços nas tarefas de coleta e organização de dados sobre a saúde, caso do DATASUS, sistema que reúne uma série de informações sobre doenças e agravos à saúde, bem como dados sobre mortalidade e morbidade, internações, infraestrutura do sistema de saúde etc. Não por acaso, este é o sistema de informações e dados mais utilizado pelos autores como fonte de indicadores de saúde.

Adicionalmente, o pesquisador envolvido com esse campo de estudos, encontra à disposição outras fontes de informações para tratar

Tabela 3. Abordagem de pesquisa, método, instrumento e técnicas analíticas.

\begin{tabular}{lrrlrr}
\hline & No & \multicolumn{1}{c}{$\%$} & No & $\%$ \\
\hline Abordagem & & & Instrumento de pesquisa & & \\
$\quad$ Quantitativa & 24 & 58,54 & Documentos & 26 & 63,41 \\
$\quad$ Qualitativa & 12 & 29,27 & Múltiplos & 11 & 26,83 \\
Mista & 5 & 12,20 & Outros & 4 & 9,76 \\
$\quad$ Total & 41 & 100,00 & Total & 41 & 100,00 \\
Método de pesquisa & & & Técnicas Analíticas & & \\
$\quad$ Pesquisa documental & 24 & 58,54 & Estatística descritiva & 9 & 21,95 \\
Estudo de caso & 4 & 9,76 & Estatística inferencial & 9 & 21,95 \\
Múltiplo & 4 & 9,76 & Estatística combinada & 8 & 19,51 \\
Etnografia & 1 & 2,44 & Combinações diversas & 3 & 7,32 \\
Outros & 8 & 19,51 & Outros & 7 & 17,07 \\
Total & 41 & 100,00 & Não informado & 5 & 12,20 \\
& & & Total & 41 & 100,00 \\
\hline
\end{tabular}

Fonte: Elaborado pelos autores. 
o tema, caso do Censo realizado pelo Instituto Brasileiro de Geografia e Estatística (IBGE), com um amplo conjunto de dados demográficos e socioeconômicos; e o Centro Nacional de Pesquisas Espaciais (INPE), que disponibiliza uma série de informações sobre o clima e as condições dos biomas brasileiros nas diferentes regiões do país. Assim, verifica-se como essa iniciativa de estruturação e democratização de fontes e bancos de dados diversos tem alimentado sistemas de informações mais consistentes com as propostas de estudos que se descreve aqui.

\section{Finalidade da produção nacional sobre o tema}

Nesta etapa do trabalho de revisão da produção científica nacional sobre a interação entre mudanças climáticas, mudanças produtivas e saúde, considerou-se a relação de palavras-chave nos 41 artigos selecionados nesta pesquisa. Os desafios da análise se dirigiram à tarefa de, a partir da lista de palavras-chave, que se mostrava muito difusa, definir temas a partir das citações mais recorrentes. Neste ponto, o recurso à metodologia de redes, teve um papel importante para evidenciar as relações estabelecidas entre palavras-chave, bem como para a identificação de temas conexos. Assim, ao incorporar esse recurso metodológico nesta fase de definição de temas, procedeu-se à aplicação da análise de redes por meio do software VOSviewer (Figura 2) ${ }^{14,15}$.

Os critérios de delimitação de temas foram definidos pela centralidade demonstrada pela forte associação existente entre as palavras-chave mudanças climáticas e desastres naturais (Figura 2). No que tange à relação estabelecida entre esses dois conceitos, vale destacar a forma como os estudos sobre mudanças climáticas e sua relação com a ocorrência de desastres naturais identificados neste artigo se orientam pela caracterização de dois contextos distintos: o espaço urbano e as regiões atingidas por seca, particularmente, o semiárido.

No caso dos estudos sobre desastres naturais focados no contexto urbano (descritos, essencialmente, a partir de eventos como inundações e deslizamentos), é possível entrever que a maior ocorrência deste tema na lista de palavras-chave pode ser explicada pelo grau visibilidade que esse assunto adquire na mídia e o impacto que o elevado número de vítimas fatais tem sobre a saúde da população. Ademais, mostra-se relevante nesse contexto a forma como o senso comum realiza a associação entre mudanças climáticas e desastres naturais - apesar da relação de causa e efeito não ser de fácil comprovação, como comentado anteriormente.

O destaque dos autores recai sobre o pressuposto de que o processo de urbanização caótico e a carência de projetos de planejamento territorial, próprios do contexto de desenvolvimento brasileiro, tornaram as cidades e metrópoles do país mais vulneráveis aos efeitos das mudanças climáticas, cujos indicadores são descritos, na maior parte dos casos, a partir de índices pluviométricos e de temperatura ${ }^{16-18}$.

De modo complementar, ao analisar a frequência das citações sobre os biomas, a Mata Atlântica aparece como o mais citado 17,7\% (exceção feita aos trabalhos que consideram no seu escopo o conjunto dos biomas do país, perfazendo um total de 26,83\%). Imprescindível notar a localização das principais metrópoles brasileiras, em termos demográficos e em razão da dinâmica econômica, essencialmente as regiões metropolitanas do Rio de Janeiro e São Paulo, ambas situadas na região sudeste e no bioma Mata Atlântica, assim como o histórico de eventos extremos com consequências trágicas experimentado por estes dois grandes centros urbanos ${ }^{19}$.

Em outra direção, ao seguir as redes de palavras e suas conexões, é possível identificar as relações que se estabelecem entre os conceitos de desastres naturais, mudanças climáticas e saúde. De modo particular, observa-se que os efeitos dos desastres naturais sobre a saúde humana incluem consequências imediatas e outras secundárias, que podem ser classificadas a partir de um amplo espectro de doenças e agravos à saúde, que ultrapassam os altos níveis de mortalidade que são decorrentes do evento em si (caso dos soterramentos e afogamentos que são comuns em situações de inundações e deslizamentos). Observa-se como os artigos analisados mostram indícios sobre o modo como os desastres naturais repercutem ao longo do tempo. Essa constatação é evidenciada pelas associações que ligam o episódio do desastre ao aumento das taxas de morbimortalidade por doenças infecciosas e crônico-degenerativas ${ }^{17,19,20}$.

Neste aspecto, que considera os efeitos secundários dos desastres naturais no contexto urbano, é possível identificar mudanças que podem ser observadas nos padrões e frequência das doenças infecciosas, particularmente, daquelas transmitidas por vetores e por veiculação hídrica (dengue, chicungunha, malária, diarreias, leptospirose etc.). Evidencia-se ainda a forma como as mudanças climáticas desempenham um papel expressivo 


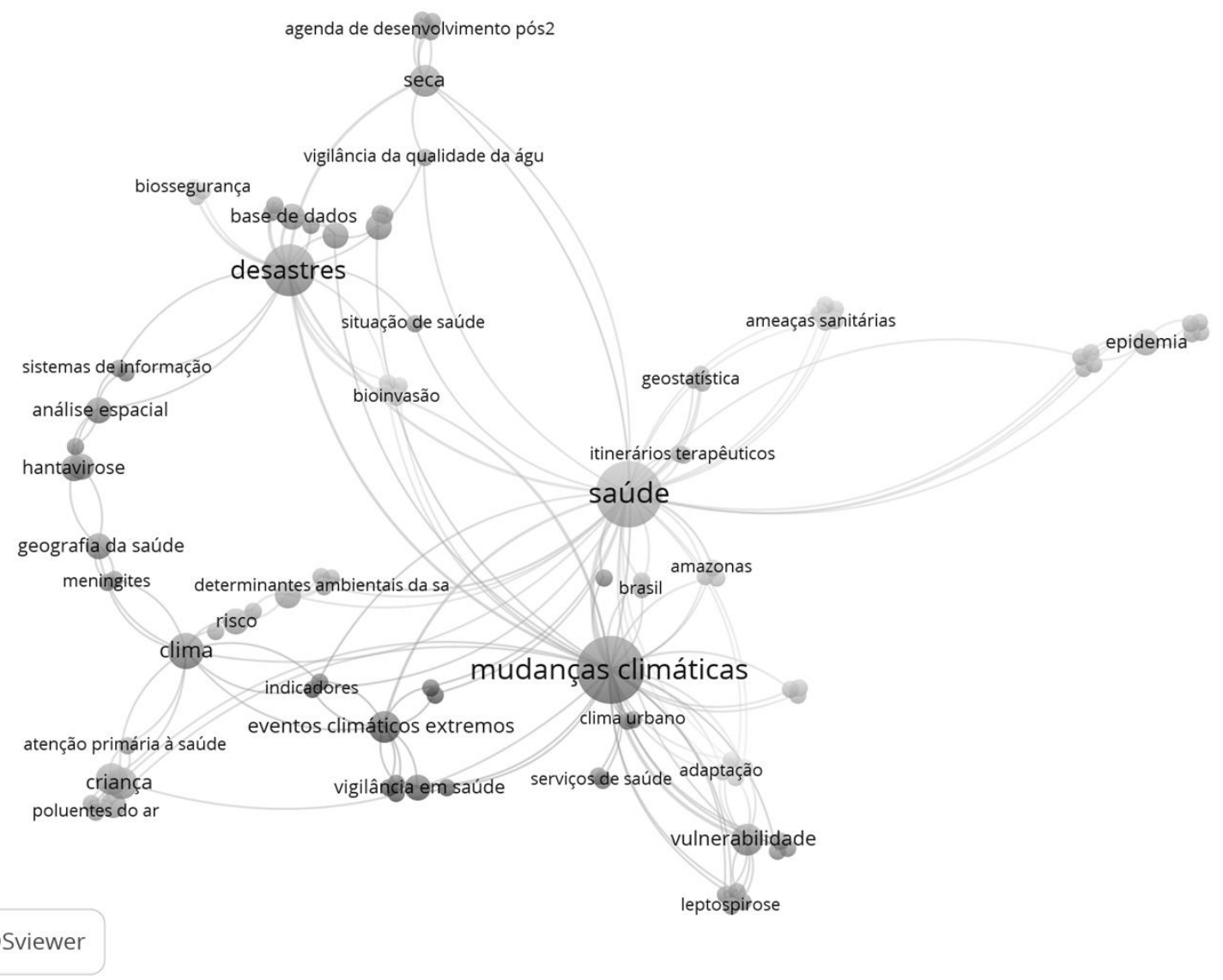

Figura 2. Rede de palavras-chave.

Fonte: Elaborado pelos autores.

na transformação do padrão de reprodução dos agentes patológicos responsáveis pelo surgimento e proliferação das doenças infecciosas.

Como apontam alguns dos estudos elegidos neste artigo, a análise dos contextos pós desastres exige a consideração de efeitos menos evidentes, cuja relação é mais difícil de ser estabelecida, a exemplo de circunstâncias em que se verifica um aumento no registro das taxas de doenças de origem crônico-degenerativa que, na maior parte dos casos, são potencializadas por situações de estresse pós-traumático (AVC, por exemplo) ${ }^{17,18,20}$.

$\mathrm{O}$ segundo grupo de estudos identificado por essa revisão da literatura relaciona o tema dos desastres naturais com o contexto das recorrentes secas que afetam a região do semiárido e em regiões caracterizadas pelo predomínio dos biomas cerrado e caatinga (citados, respectivamente, por
12,20 e 7,32\% dos artigos compilados no presente trabalho). Para além da identificação das vulnerabilidades ambientais, o tema é tratado pelos autores a partir do foco nas vulnerabilidades socioeconômicas e pela fragilidade institucional e de governança que caracterizam algumas regiões do país ${ }^{21,22}$.

No que concerne ao aspecto da saúde, assume proeminência o papel de um sistema falho de saneamento básico e uma situação crônica de insegurança alimentar e hídrica, para a ocorrência de doenças e agravos à saúde. Não por acaso, as maiores taxas de mortalidade infantil por diarreia e por agravos à saúde decorrentes da subnutrição e da má distribuição de água potável estão concentradas nesta região do país. Dessa informação é possível compreender a importância atribuída pelos autores às doenças infecciosas de 
veiculação hídrica, pelo consumo por alimentos contaminados ou por vetores.

De fato, a região Nordeste do país é apontada como a mais vulnerável pelo conjunto dos estudos analisados ${ }^{13,22-25}$, quando considerados os efeitos das mudanças climáticas, cujas implicações negativas não se restringem ao modo como afetam as condições de saúde da população, mas atingem de forma drástica os sistemas produtivos, em especial, a agricultura.

Logo, são nas pesquisas focadas no contexto do semiárido que a influência operada pelas mudanças climáticas e sua interface com as mudanças produtivas se mostrou mais realçada. Embora o tema de mudanças produtivas apareça de forma tangencial em muitas das investigações consideradas, serão nos estudos sobre o semiárido e sobre a região do Nordeste do país, que esse tema será tratado de forma mais atenta e sistemática. Esse dado confirma a relevância de se focalizar esta região para a produção de conhecimento relevante sobre as vulnerabilidades que se apresentam, assim como no necessário desenvolvimento de dispositivos de adaptação por parte das populações expostas aos riscos relacionados aos efeitos das mudanças climáticas.

\section{O caleidoscópio de dados e informações: indicadores de mudanças climáticas, mudanças produtivas e saúde e suas respectivas bases de dados}

A incursão na complexidade que encerra os estudos produzidos sobre as interações que podem ser observadas entre mudanças climáticas, mudanças produtivas e saúde possibilitou o aprofundamento do trabalho de revisão da literatura, no sentido de ampliar o escopo da investigação para o mapeamento dos indicadores mais referidos pelos autores na composição de suas análises. Assim, neste ponto, tratar-se-á dos indicadores de mudanças climáticas, mudanças produtivas e saúde acionados pelos autores, bem como da problematização do conceito de vulnerabilidade, como recurso complementar à caracterização temática dos artigos.

Considera-se que esta tarefa não se restringiu à apresentação dos elementos acionados para a compreensão do assunto em tela, mas possibilitou a identificação das fontes dos dados e informações apresentados pelos pesquisadores (Quadro 1). Acredita-se que o mapeamento de indicadores e suas bases de dados disponibiliza um recurso adicional às pesquisas que podem vir a ser produzidas sobre o tema, ao mesmo tempo em que se mostra como um parâmetro interessante que deve auxiliar na identificação de lacunas de investigação que poderão mais facilmente ser superadas no futuro.

\section{Os efeitos do clima sobre o padrão epidemiológico}

Destaca-se a contribuição dos estudos focalizados neste artigo, ao demonstrarem como, na atualidade, é possível identificar mudanças no padrão epidemiológico que, em muitos casos, se vê afetado pelas mudanças climáticas ${ }^{26}$. De fato, essa tendência demonstrada por estudos mais recentes, evidencia o esforço de alguns pesquisadores em criar instrumentos e modelos de análise até então pouco utilizados pela epidemiologia. A este respeito, merece atenção o uso de ferramentas de Sistemas de Informações Geográficas (SIG) entre outros modelos de análise que permitem, a partir do inter-relacionamento entre dados ambientais e de saúde, a identificação e quantificação de populações sujeitas a riscos ${ }^{27}$.

Os resultados obtidos a partir do uso de recursos desse tipo podem ser observados em diferentes artigos analisados neste trabalho ${ }^{13,23,26,28} \mathrm{e}$ demonstram que para além da identificação das populações sujeitas a risco, os avanços nessa área permitem prover sistemas de diagnóstico, modelagem, análise e intervenção apropriados aos contextos mais suscetíveis às mudanças climáticas. Ademais, avançam ao fornecer aportes importantes à tomada de decisão e ao planejamento de políticas públicas.

Por fim, em relação à análise dos indicadores de saúde mencionados pelos autores nos artigos analisados, os resultados mostram o destaque dado às doenças infecciosas, conforme aventado anteriormente, especialmente, àquelas transmitidas por veiculação hídrica, por alimento contaminado ou por vetores (dengue, leishmaniose, leptospirose, chicungunha, malária, diarreia etc.), sendo descritas em 39,02\% dos artigos, com menor destaque para aquelas de origem ambiental $(17,07 \%)$ e outras de origem crônico-degenerativas $(4,88 \%)$.

\section{A interface entre mudanças climáticas e mudanças produtivas}

Em relação aos efeitos das mudanças climáticas e sua influência sobre a capacidade produtiva, especialmente no que tange ao uso do solo e os sistemas agrícolas, os resultados do mapeamento dos indicadores de mudanças produtivas eviden- 
Quadro 1. Principais indicadores para cada tema (saúde, mudanças climáticas e produtivas).

\begin{tabular}{|c|c|c|}
\hline Área & Indicadores & Bases de dados \\
\hline Saúde & $\begin{array}{l}\text { Taxa de incidência de doenças } \\
\text { Taxa de morbidade } \\
\text { Taxa de mortalidade } \\
\text { Número de internações } \\
\text { Custo das internações } \\
\text { População com plano de saúde } \\
\text { Danos materiais ao Setor de Saúde Pública } \\
\text { Investimentos no Setor de Saúde Pública }\end{array}$ & $\begin{array}{l}\text { Departamento de Informática do Sistema Único de } \\
\text { Saúde (DATASUS) } \\
\text { Atlas do Desenvolvimento Humano no Brasil } \\
\text { (PNUD) } \\
\text { Censo Brasileiro (IBGE) } \\
\text { Sistema de Informação de Agravos de Notificação } \\
\text { (SINAN) } \\
\text { Superintendências Regionais de Saúde } \\
\text { Secretarias Estaduais e Municipais de Saúde } \\
\text { Observatório Nacional de Clima e Saúde }\end{array}$ \\
\hline $\begin{array}{l}\text { Mudanças } \\
\text { climáticas }\end{array}$ & $\begin{array}{l}\text { Desastres naturais } \\
\text { Precipitação } \\
\text { Temperatura } \\
\text { Umidade } \\
\text { Pressão atmosférica } \\
\text { Velocidade do vento } \\
\text { Focos de queimadas } \\
\text { Monóxido de carbono } \\
\text { Dióxido de enxofre } \\
\text { Dióxido de nitrogênio } \\
\text { Ozônio } \\
\text { Irradiância espectral da UV } \\
\text { Índice UV } \\
\text { Radiação solar global } \\
\text { Espessura óptica de aerossol }\end{array}$ & $\begin{array}{l}\text { Instituto Nacional de Pesquisas Espaciais (INPE) } \\
\text { Instituto Nacional de Meteorologia (INMET) } \\
\text { Agência Nacional de Águas (ANA) } \\
\text { Centros Estaduais e Municipais de Meteorologia } \\
\text { Secretarias Estaduais e Nacional de Defesa Civil } \\
\text { Decretos de situação de emergência e estado de } \\
\text { calamidade pública } \\
\text { Atlas Brasileiro dos Desastres Naturais } \\
\text { Painel Brasileiro de Mudanças Climáticas (PBMC) } \\
\text { Sistema de Informações Ambientais (SISAM) } \\
\text { Centro Regional de Informações Sobre Desastres } \\
\text { (CRID) } \\
\text { International Panel onClimate Change (IPCC) } \\
\text { Observatório Nacional de Clima e Saúde }\end{array}$ \\
\hline $\begin{array}{l}\text { Mudanças } \\
\text { produtivas }\end{array}$ & $\begin{array}{l}\text { Base cartográfica digital do uso e da } \\
\text { cobertura da terra } \\
\text { Atividades de agropecuárias } \\
\text { Taxa de desmatamento }\end{array}$ & $\begin{array}{l}\text { Instituto Brasileiro de Geografia e Estatística } \\
\text { (IBGE) } \\
\text { Ministério do Meio Ambiente (MMA) }\end{array}$ \\
\hline
\end{tabular}

Fonte: Elaborado pelos autores.

ciaram que este tema, mesmo que não tenha recebido um destaque especial nas pesquisas consideradas, mostrou-se como questão recorrente em um número significativo de artigos.

Entre os indicadores acionados pelos autores para a descrição das mudanças produtivas merecem destaque aqueles relacionados ao uso da cobertura da terra e as taxas desmatamento, no bioma cerrado ${ }^{29}$ e na região da Amazônia ${ }^{30,31}$.

\section{O caráter multidimensional da vulnerabilidade humana}

Como mencionado, a relação entre mudanças climáticas, mudanças produtivas e saúde humana implica a compreensão das vulnerabilidades e riscos aos quais as populações humanas estão expostas, cujo desenvolvimento teórico alcançou ao longo das últimas décadas um incremento considerável, pela forma como esses conceitos integram questões biofísicas, socioeconômicas, culturais e institucionais ${ }^{4-6}$.

No conjunto de artigos analisados, as transformações dos parâmetros teóricos que orientam as pesquisas podem ser evidenciadas de modo mais acentuado no deslocamento da ênfase na ideia de mitigação (caso do efeito estufa, por exemplo) para propostas de análises centradas no conceito de adaptação, fundamental para a proposição de políticas públicas orientadas aos cenários e desafios apresentados pelas mudanças climáticas na atualidade ${ }^{13,22}$.

Consequentemente, observa-se que a menção ao conceito de vulnerabilidade e seu caráter multidimensional é registrada em 51,22\% dos artigos coligidos neste estudo. Em segundo lugar, é mencionado o aspecto ambiental deste conceito $(24,39 \%)$, seguido das dimensões econômica $(7,32 \%)$, social $(4,88 \%)$ e institucional $(2,44 \%)$. 


\section{Considerações finais}

A análise do conjunto da produção científica nacional sobre a interação entre mudanças climáticas, produtivas e saúde humana demonstrou o impulso que nos últimos dez anos o desenvolvimento de pesquisas de banco de dados e informações integradas conferiu aos estudos interdisciplinares produzidos sobre este tema. A este respeito, destaca-se como se realizou o aperfeiçoamento de ferramentas e instrumentos analíticos, capazes de reunir informações consistentes sobre uma realidade complexa e ampla, marcada pela diversidade dos biomas, sistemas produtivos e diferentes contextos socioeconômicos que o país oferece.

Em outra direção, conquanto seja possível destacar os aportes para a consolidação de bancos de dados e informações que surgem como resultado do processo de democratização experimentado nos últimos anos com a criação de agências públicas, como o DATASUS, as pesquisas mostram que incursões nas pesquisas empíricas ainda são reduzidas, com forte participação de investigações centradas em dados secundários.

Com efeito, quando considerado os avanços obtidos no campo teórico-metodológico, verifica-se que estes se revelam tímidos na medida em que a ênfase em métodos de análise ocupou espaço privilegiado nas agendas de pesquisa. Entretanto, há de notar a centralidade do conceito de vulnerabilidade, cujo rendimento se mostrou relevante para o entendimento da complexidade das interações existentes. No que tange à proble- matização dos efeitos das mudanças climáticas e sua influência sobre a capacidade produtiva, este tema se mostrou secundário, apesar de se mostrar relevante ao indicar caminhos promissores para o desenvolvimento de novas pesquisas.

O conjunto da literatura analisada neste trabalho revela, ainda, a importância da pesquisa sobre o tema para a produção de subsídios destinados à formulação de políticas públicas, essencialmente, pela necessidade de dotar os serviços de saúde de recursos adequados à identificação de vulnerabilidades e antecipação de situações de risco, centradas no desenvolvimento de capacidades adaptativas aos problemas relacionados às mudanças climáticas. O próximo passo seria, então, acompanhar como os avanços apresentados nesses trabalhos têm repercutido na pauta dos gestores públicos.

Por fim, no que tange às redes de pesquisa, verifica-se a importância do papel das equipes multidisciplinares e o circuito colaborativo de instituições de pesquisa e órgãos públicos, característica que se mostra afinada com aquela obtida a partir da análise da produção internacional sobre o tema ${ }^{11}$. Logo, é possível destacar a existência de um perfil profissional cuja formação se concentra nas áreas de Geografia, Ciências Biológicas e Engenharias, com participação ainda muito reduzida de profissionais nas áreas médicas o que revela, por sua vez, a vocação de tratar o tema de forma intersetorial e colaborativa, com capacidade de mobilizar diferentes campos da pesquisa científica. 


\section{Colaboradores}

L Santos se dedicou às tarefas de delineamento da pesquisa, execução do projeto inicial, coleta e análise dos dados, assim como à redação final do artigo. JE Oliveira trabalhou na coleta e análise dos dados, elaboração das tabelas e gráficos, descrição da metodologia, redação e revisão final do texto. Na condição de Coordenadores do Projeto, A Maduro-Abreu e G Litre foram responsáveis pela concepção da ideia original do artigo, definição teórico-metodológica, revisão crítica e aprovação final do manuscrito. Em relação à tarefa de produção de gráfico para a análise de redes, a partir do software VOSviewer, esta foi conduzida por GS Sátiro e DC Soares, que também forneceram informações e dados para a realização da análise comparativa entre os resultados obtidos e o estado da arte da literatura internacional sobre o tema focalizado no artigo.

\section{Referências}

1. World Health Organization (WHO). Potential health effects of climatic change. Genebra: WHO; 1990.

2. Organização Mundial de Saúde (OMS). Mudança climática e saúde humana: riscos e respostas: resumo atualizado. Brasília: OPAS/OMS; 2008.

3. Organização Pan-Americana da Saúde (OPAS). Mudança climática e saúde: um perfil do Brasil. Brasília: OPAS/MS; 2009.

4. Adger WN. Vulnerability. Glob Environ Change 2006; 16:268-281.

5. O'Brien KL, Eriksen S, Sygna L, Naess L. O. Questioning complacency: climate change impacts, vulnerability, and adaptation in Norway. AMBIO 2006; 35(2):50-56.

6. Hogan DJ, Mandarola Jr. E. Towards an interdisciplinary conceptualisation of vulnerability. Popul Space Place 2005; 11:455-471.

7. Guarnieri P, Sobreiro VA, Nagano MS, Marques Serrano AL. The challenge of selecting and evaluating third-party reverse logistics providers in a multicriteria perspective: Brazilian case. J Clean Prod 2015; 96:209-219.

8. Furie GL, Balbus J. Global environmental health and sustainable development: the role at Rio+20. Cien Saude Colet 2012; 17(6):1427-1432.

9. Carvalho BM, Rangel EF, Vale MM. Evaluation of the impacts of climate change on disease vectors through ecological niche modelling. Bull Entomol Res 2017; 107(4):419-430.

10. Correa-Macana E, Comim F. Mudança climática e desenvolvimento humano: uma análise baseada na Abordagem das Capacitações de Amartya Sen. Econ Soc $y$ Territ 2013; 13(43):577-618.

11. Maduro-Abreu A, Sátiro GS, Soares DC, Litre G, Santos L, Oliveira JE. A interface entre saúde, mudanças climáticas e o uso do solo no Brasil: uma revisão sistemática da produção científica internacional entre 1993 e 2018. Saude Soc 2020; 29(2):e180866.

12. Elsevier. Gender in the Global Research Landscape. Analysis of research performance through a gender lens across 20 years, 12 geographies, and 27 subject areas [Internet]. 2017 [acessado 2018 maio 18]. Disponível em: https://www.elsevier.com/_data/assets/pdf_file /0008/265661/ElsevierGenderReport_final_for-web. pdf.

13. Barcellos C, Monteiro AMV, Corvalán C, Gurgel HC, Carvalho MS, Artaxo P, Hacon S, Ragoni V. Mudanças climáticas e ambientais e as doenças infecciosas: cenários e incertezas para o Brasil. Epidemiol Serv Saude 2009; 18(3):285-304.

14. Van Eck NJ, Waltman L. How to normalize co-occurrence data? An analysis of some well-known similarity measures. J Am Soc Inf Sci Technol 2009; 60(8):16351651.

15. Waltman L, Van Eck NJ. A smart local moving algorithm for large-scale modularity-based community detection. Eur Phys J B 2013; 86(471):1-14.

16. Cardoso TAO. Biossegurança e desastres: conceitos, prevenção, saúde pública e manejo de cadáveres. Physis 2012; 22(4):1523-1542. 
17. Freitas CM, Silva DRX, Sena ARM, Silva EL, Sales LBF, Carvalho ML, Mazoto ML, Barcellos C, Costa AM, Oliveira MLC, Corvalán C. Desastres naturais e saúde: uma análise da situação do Brasil. Cien Saude Colet 2014; 19(9):3645-3656.

18. Xavier DR, Barcellos C, Barros HS, Magalhães MAFM, Matos VP, Pedroso MM. Organização, disponibilização e possibilidades de análise de dados sobre desastres de origem climática e seus impactos sobre a saúde no Brasil. Cien Saude Colet 2014; 19(9):3657-3668.

19. Oliveira TVS. Variáveis climáticas, condições de vida e saúde da população: a leptospirose no Município do Rio de Janeiro de 1996 a 2009. Cien Saude Colet 2012; 17(6):1569-1576.

20. Pereira CAR, Barata MML, Hoelz MPC, Medeiros VNLO, Marincola FCV, Costa Neto C, Marinho DP, Oliveira TVS, Trigo AGM, Medeiros TK. Avaliação econômica dos casos de dengue atribuídos ao desastre de 2011 em Nova Friburgo (RJ), Brasil. Cien Saude Colet 2014; 19(9):3693-3704.

21. Alpino TA, Sena ARM, Freitas CM. Desastres relacionados à seca e saúde coletiva - uma revisão da literatura científica. Cien Saude Colet 2016; 21(3):809-820.

22. Centro de Desenvolvimento e Planejamento Regional (CEDENPLAR). Fundação Oswaldo Cruz (Fiocruz). Mudanças climáticas, migrações e saúde: cenários para $o$ nordeste brasileiro 2000-2050. Belo Horizonte: CEDEPLAR/UFMG/Fiocruz; 2008.

23. Queiroz BL, Barbieri AF, Confalonieri UE. Mudanças climáticas, dinâmica demográfica e saúde: desafios para o planejamento e as políticas públicas no Brasil. Rev Polit Planeja Regi 2016; 3(1):93-116.

24. Barbieri AF. Mudanças climáticas, mobilidade populacional e cenários de vulnerabilidade para o Brasil. Rev Inter Mob Hum 2011; XIX(36):95-112.

25. Sena ARM, Freitas CM, Barcellos C, Ramalho V, Corvalan C. Medindo o invisível: análise dos Objetivos de Desenvolvimento Sustentável em populações expostas à seca. Cien Saude Colet 2016; 21(3):671-684.
26. Lima-Camara TN. Arboviroses emergentes e novos desafios para a saúde pública no Brasil. Rev Saude Publica 2016; 50:36.

27. Barcellos C, Coutinho K, Pina MDF, Magalhães MMAF, Paola JCMD, Santos SM. Inter-relacionamento de dados ambientais e de saúde: análise de risco à saúde aplicada ao abastecimento de água no Rio de Janeiro utilizando Sistemas de Informações Geográficas. Cad Saude Publica 1998; 14(3):597-605.

28. Rufino R, Gracie R, Sena A, Freitas CM, Barcellos C. Surtos de diarreia na região Nordeste do Brasil em 2013, segundo a mídia e sistemas de informação de saúde - Vigilância de situações climáticas de risco e emergências em saúde. Cien Saude Colet 2016; 21(3):777-788.

29. Santos J, Oliveira SV, Steinke VA. O uso e a cobertura da terra e a sua relação com a hantavirose na região integrada de desenvolvimento do Distrito Federal e entorno. Raega 2016; 3(7):282-306.

30. Santos MRS. Vulnerabilidade e mudanças climáticas: análise socioambiental em uma mesorregião da Amazônia. Rev Ambient Água 2017; 12(5):842-854.

31. Viana VM. Bolsa Floresta um instrumento inovador para a promoção da saúde em comunidades tradicionais na Amazônia. Estud Av 2008; 22(64):143-153.

Artigo apresentado em 04/09/2018

Aprovado em 01/11/2019

Versão final apresentada em 03/11/2019

Editores-chefes: Romeu Gomes, Antônio Augusto Moura da Silva 